DOI: 10.2478/ausm-2014-0002

\title{
Some inequalities in bicentric quadrilateral
}

\author{
Mihály Bencze \\ 505600 Săcele-Négyfalu, \\ Jud. Braşov, Romania \\ email: benczemihaly@gmail.com
}

\author{
Marius Drăgan \\ 061311 bd. Timişoara nr. 35, \\ bl. OD6, sc. E, et. 7 ap. 176 , Sect. 6 ., \\ Bucureşti, Romania \\ email: marius.dragan2005@yahoo.com
}

Dedicated to the memory of Professor Antal Bege

\begin{abstract}
In this paper we prove some results concerning bicentric quadrilaterals. We offer a new proof of the Blundon-Eddy inequality, which we use to obtain other inequalities in bicentric quadrilaterals.
\end{abstract}

\section{Introduction}

Let $A B C D$ be a bicentric quadrilateral with $a=A B, b=B C, c=C D, d=$ $A D, d_{1}=A C, d_{2}=B D, s=\frac{a+b+c+d}{2}, R$ the radius of the circumscribed circle of the quadrilateral $A B C D$ and $r$ the radius of the inscribed circle, $F$ the area.

In [1] W. J. Blundon and R. H. Eddy proved that:

$$
8 r\left(\sqrt{4 R^{2}+r^{2}}-r\right) \leq s^{2} \leq(r+\sqrt{4 R+r})^{2} .
$$

In the following we give a simple proof to this double inequality using the product

$$
(a-b)^{2}(a-c)^{2}(a-d)^{2}(b-c)^{2}(b-d)^{2}(c-d)^{2},
$$

then we deduce many other important new inequalities. We mention that the result concerning the above product is new.

We denote:

$\sigma_{1}=\sum a, \sigma_{2}=\sum a b, \sigma_{3}=\sum a b c, x_{1}=b c+a d, x_{2}=a b+c d, x_{3}=a c+b d$. 


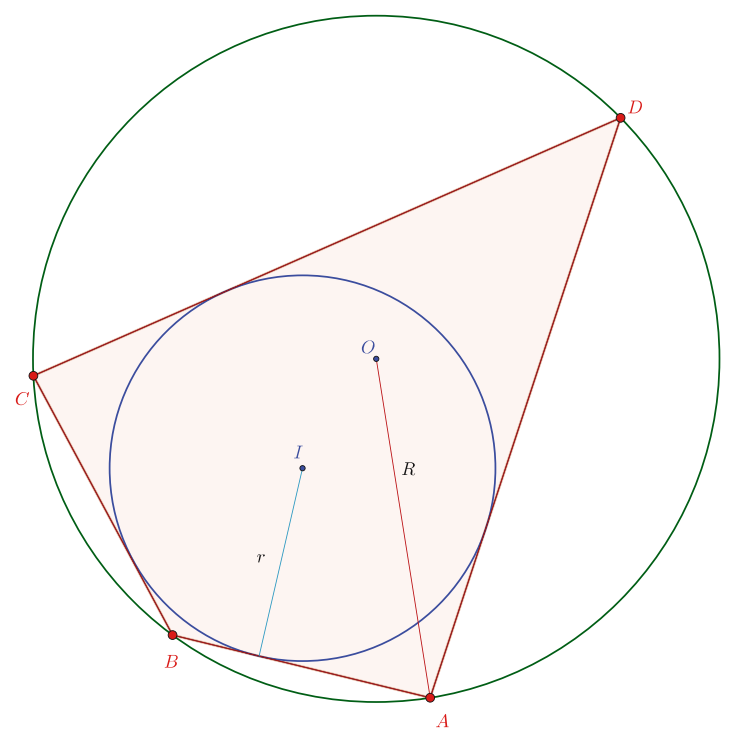

\section{Main results}

Lemma 1 In every bicentric quadrilateral ABCD the following equalities are true:

1) $F^{2}=(s-a)(s-b)(s-c)(s-d)=a b c d ;$

2) $x_{1} x_{2} x_{3}=16 R^{2} r^{2} s^{2}$

3) $x_{1}+x_{2}=s^{2}$

4) $x_{1}+x_{2}+x_{3}=s^{2}+2 r^{2}+2 r \sqrt{r^{2}+4 R^{2}}$;

5) $x_{3}=2 r\left(r+\sqrt{4 R^{2}+r^{2}}\right)$;

6) $(a-b)^{2}(a-c)^{2}(a-d)^{2}(b-c)^{2}(b-d)^{2}(c-d)^{2}=\left(x_{1}-x_{2}\right)^{2}\left(x_{2}-x_{3}\right)^{2}$ $\left(x_{3}-x_{1}\right)^{2}$.

\section{Proof.}

1) We have $a+c=b+d$. It results that $s-b=d$ and three similar equalities which imply

$$
(s-a)(s-b)(s-c)(s-d)=a b c d .
$$

2) From Ptolemy's theorem it results that $x_{3}=d_{1} d_{2}$. We have the equalities:

$$
a d \sin A+b c \sin C=2 F, a b \sin B+d c \sin D=2 F
$$


We obtain $(a d+b c) d_{1}=4 R F,(a b+d c) d_{2}=4 R F$ which implies

$$
(a d+b c)(a b+d c) d_{1} d_{2}=16 R^{2} F^{2} \text { or } x_{1} x_{2} x_{3}=16 R^{2} r^{2} s^{2} .
$$

3) We have $x_{1}+x_{2}=a d+b c+a b+c d=(a+c)(d+b)=(a+c)^{2}=$ $\left(\frac{a+b+c+d}{2}\right)=s^{2}$.

4) From (1) it results that

$$
\begin{aligned}
& (a b+b c)(a d+d c)(a c+b d)=16 R^{2} F^{2} \text { or } \\
& a b c d \sum a^{2}+\sigma_{3}^{2}-2 a b c d \sigma_{2}=16 R^{2} F^{2} \text { or } \\
& \sigma_{3}^{2}-4 s^{2} r^{2} \sigma_{2}+4 s^{4} r^{2}=16 R^{2} r^{2} s^{2} v .
\end{aligned}
$$

But $(s-a)(s-b)(s-c)(s-d)=s^{2} r^{2}$ or $-s^{3}+\sigma_{2} s-\sigma_{3}=0$ which implies

$$
\sigma_{3}=s\left(\sigma_{2}-s^{2}\right)
$$

From (2) and (3) we have:

$$
\begin{aligned}
& s^{2}\left(\sigma_{2}-s^{2}\right)^{2}-4 s^{2} r^{2} \sigma_{2}+4 s^{4} r^{2}=16 R^{2} r^{2} s^{2} \quad \text { or } \\
& \sigma_{2}^{2}-\left(2 s^{2}+4 r^{2}\right) \sigma_{2}+s^{4}+2 s^{2} r^{2}-16 r^{2} R^{2}=0 .
\end{aligned}
$$

It results that: $\sigma_{2}=s^{2}+2 r^{2}+2 r \sqrt{r^{2}+4 R^{2}}$. But $\sigma_{2}=x_{1}+x_{2}+x_{3}$, so it follows that

$$
x_{1}+x_{2}+x_{3}=s^{2}+2 r^{2}+2 r \sqrt{r^{2}+4 R^{2}} .
$$

5) From 4) since $x_{1}+x_{2}=s^{2}$ it follows that $x_{3}=2 r^{2}+2 r \sqrt{4 R^{2}+r^{2}}$.

6) We have $(a-b)^{2}(a-c)^{2}(a-d)^{2}(b-c)^{2}(b-d)^{2}(c-d)^{2}=$ $[(a-b)(c-d)]^{2}[(a-c)(b-d)]^{2}[(a-d)(b-c)]^{2}=$ $\left(x_{1}-x_{2}\right)^{2}\left(x_{2}-x_{3}\right)^{2}\left(x_{2}-x_{1}\right)^{2}$.

Theorem 1 In every bicentric quadrilateral ABCD the following equality is true:

$$
\begin{aligned}
& (a-b)^{2}(a-c)^{2}(a-d)^{2}(b-c)^{2}(b-d)^{2}(c-d)^{2} \\
& =16 r^{4} s^{2}\left[s^{2}-8 r\left(\sqrt{4 R^{2}+r^{2}}-r\right)\right]\left[s^{2}-\left(r+\sqrt{4 R^{2}+r^{2}}\right)^{2}\right]^{2} .
\end{aligned}
$$


Proof. We denote $\Delta=(a-b)^{2}(a-c)^{2}(a-d)^{2}(b-c)^{2}(b-d)^{2}(c-d)^{2}$. From Lemma 1 6) we have:

$$
\begin{aligned}
\triangle & =\left(x_{1}-x_{2}\right)^{2}\left(x_{3}-x_{1}\right)^{2}\left(x_{3}-x_{2}\right)^{2} \\
& =\left[\left(x_{1}+x_{2}\right)^{2}-4 x_{1} x_{2}\right]\left[x_{3}^{2}-x_{3}\left(x_{1}+x_{2}\right)+x_{1} x_{2}\right]^{2} .
\end{aligned}
$$

From Lemma 12 ) and 5) it results that:

$$
x_{1} x_{2}=\frac{8 R^{2} r^{2} s^{2}}{r\left(r+\sqrt{4 R^{2}+r^{2}}\right)}=2 r\left(\sqrt{4 R^{2}+r^{2}}-r\right) s^{2} .
$$

From Lemma 1 3), 5) and equalities (5), (6) we obtain:

$$
\begin{aligned}
\triangle= & {\left[s^{4}-8 r\left(\sqrt{4 R^{2}+r^{2}}-r\right) s^{2}\right]\left[4 r^{2}\left(r+\sqrt{4 R^{2}+r^{2}}\right)^{2}\right.} \\
& \left.-2 s^{2} r\left(r+\sqrt{4 R^{2}+r^{2}}\right)+2 r\left(\sqrt{4 R^{2}+r^{2}}-r\right) s^{2}\right]^{2} \\
= & s^{2}\left[s^{2}-8 r\left(\sqrt{4 R^{2}+r^{2}}-r\right)\right]\left[4 r^{2}\left(r+\sqrt{4 R^{2}+r^{2}}\right)^{2}-4 r^{2} s^{2}\right]^{2} \\
= & 16 r^{4} s^{2}\left[s^{2}-8 r\left(\sqrt{4 R^{2}+r^{2}}-r\right)\right]\left[s^{2}-\left(r+\sqrt{4 R^{2}+r^{2}}\right)^{2}\right] .
\end{aligned}
$$

Theorem 2 In every bicentric quadrilateral ABCD the following double inequality is true: $8 \mathrm{r}\left(\sqrt{4 \mathrm{R}^{2}+\mathrm{r}^{2}}-\mathrm{r}\right) \leq \mathrm{s}^{2} \leq\left(\mathrm{r}+\sqrt{4 \mathrm{R}^{2}+\mathrm{r}^{2}}\right)^{2}$. The equality holds in the case of two bicentric quadrilaterals $\mathrm{A}_{1} \mathrm{~B}_{1} \mathrm{C}_{1} \mathrm{D}_{1}$ and $\mathrm{A}_{2} \mathrm{~B}_{2} \mathrm{C}_{2} \mathrm{D}_{2}$ with the sides

$$
\begin{aligned}
& a_{1}=c_{1}=\sqrt{2 r \sqrt{4 R^{2}+r^{2}}-2 r^{2}} \\
& b_{1}=\sqrt{2 r \sqrt{4 R^{2}+r^{2}}-2 r^{2}}-\sqrt{2 r \sqrt{4 R^{2}+r^{2}}-6 r^{2}} \\
& d_{1}=\sqrt{2 r \sqrt{4 R^{2}+r^{2}}-2 r^{2}}+\sqrt{2 r \sqrt{4 R^{2}+r^{2}}-6 r^{2}} \\
& a_{2}=d_{2}=\frac{r+\sqrt{r^{2}+4 R^{2}}-\sqrt{4 R^{2}-2 r^{2}-2 r \sqrt{4 R^{2}+r^{2}}}}{2} \\
& b_{2}=c_{2}=\frac{r+\sqrt{r^{2}+4 R^{2}}+\sqrt{4 R^{2}-2 r^{2}-2 r \sqrt{4 R^{2}+r^{2}}}}{2} .
\end{aligned}
$$


Proof. We have $\left(x_{3}-x_{1}\right)\left(x_{3}-x_{2}\right)=(a-b)(b-c)(c-d)(d-a)$ and because $a+c=b+d$ it results that $(a-b)(b-c)(c-d)(d-a)=(a-b)^{2}$ $(b-c)^{2} \geq 0$, which implies $\left(x_{3}-x_{1}\right)\left(x_{3}-x_{2}\right) \geq 0$ or

$$
s^{2} \leq\left(r+\sqrt{4 R^{2}+r^{2}}\right)^{2} .
$$

But, from Theorem 1 since $\triangle \geq 0$, it results that

$$
8 r\left(\sqrt{4 R^{2}+r^{2}}-r\right) \leq s^{2} .
$$

It remain to study the equality cases for $s_{1} \leq s \leq s_{2}$ where

$$
s_{1}=\sqrt{8 r\left(\sqrt{4 R^{2}+r^{2}}-r\right)}, s_{2}=r+\sqrt{4 R^{2}+r^{2}} .
$$

From Theorem 1 it results that we may have the cases:

\section{Case 1.}

$$
a=c .
$$

We denote $a=x$. Then

$$
a=x, b=y, c=x, d=2 x-y
$$

From Lemma 1 we have:

$$
x_{3}=2 r\left(r+\sqrt{4 R^{2}+r^{2}}\right) \text { or } x^{2}+y(2 x-y)=2 r\left(r+\sqrt{4 R^{2}+r^{2}}\right) .
$$

But $F^{2}=\operatorname{abcd}$ or $(2 x-y) y=4 r^{2}$. It results that $x^{2}=2 r \sqrt{4 R^{2}+r^{2}}-2 r^{2}$. Since $s_{1}^{2}=4 x^{2}=8 r\left(\sqrt{4 R^{2}+r^{2}}-r\right)$ represents the left side of the inequality from the statement, so:

$$
\begin{aligned}
& x=\sqrt{2 r \sqrt{4 R^{2}+r^{2}}-2 r^{2}} \\
& (y-x)^{2}=2 r \sqrt{4 R^{2}+r^{2}}-6 r^{2} \text { or }|y-x|=\sqrt{2 r \sqrt{4 R^{2}+r^{2}}-6 r^{2}} .
\end{aligned}
$$

We denote $u_{1}=2 r \sqrt{4 R^{2}+r^{2}}-2 r^{2}, u_{2}=2 r \sqrt{4 R^{2}+r^{2}}-6 r^{2}$.

If $x \leq y$ we have

$$
a=x=\sqrt{u_{1}}, b=y=\sqrt{u_{1}}+\sqrt{u_{2}}, c=\sqrt{u_{1}}, d=2 x-y=\sqrt{u_{1}}-\sqrt{u_{2}} .
$$


If $x>y$ we have

$a=x=\sqrt{u_{1}}, b=y=x-\sqrt{u_{2}}=\sqrt{u_{1}}-\sqrt{u_{2}}, c=\sqrt{u_{1}}, d=2 x-y=\sqrt{u_{1}}+\sqrt{u_{2}}$.

It results that the equality from the left side of the inequality of the statement holds in the case of bicentric quadrilateral $A_{1} B_{1} C_{1} D_{1}$ with the sides

$$
\sqrt{u_{1}}, \sqrt{u_{1}}-\sqrt{u_{2}}, \sqrt{u_{1}}, \sqrt{u_{1}}+\sqrt{u_{2}} .
$$

\section{Case 2.}

$$
\mathrm{a}=\mathrm{d}=\mathrm{x}, \mathrm{b}=\mathrm{c}=\mathrm{y} .
$$

In this case $m(\measuredangle D)=m(\measuredangle B)=90^{\circ}, A C=2 R$. It results that $F=s r=2 \frac{x y}{2}$ or $x y=(x+y) r$.

We denote $\alpha=x+y, \beta=x y$.

We have $\beta=\alpha$ r. But $x^{2}+y^{2}=4 R^{2}$ which implies $\alpha^{2}-2 \beta=4 R^{2}$ so we have $\alpha^{2}-2 \alpha r-4 R^{2}=0$.

It results that $\alpha=\mathrm{r}+\sqrt{\mathrm{r}^{2}+4 \mathrm{R}^{2}}$.

But $s_{1}=x+y=\alpha=r+\sqrt{r^{2}+4 R^{2}}$ which represents the right side of the inequality from the statement. We have $\left\{\begin{array}{l}x+y=\alpha \\ x y=r \alpha\end{array}\right.$, so $x, y$ are the solutions of the equation $u^{2}-\alpha u+r \alpha=0$ which implies:

$$
\begin{aligned}
& x=\frac{\alpha-\sqrt{\alpha^{2}-4 r \alpha}}{2}=\frac{r+\sqrt{r^{2}+4 R^{2}}-\sqrt{4 R^{2}-2 r^{2}-2 r \sqrt{4 R^{2}+r^{2}}}}{2} \\
& y=\frac{r+\sqrt{r^{2}+4 R^{2}}+\sqrt{4 R^{2}-2 r^{2}-2 r \sqrt{4 R^{2}+r^{2}}}}{2} .
\end{aligned}
$$

So, the equality for the right side of the inequality from the statement is true in the case of bicentric quadrilateral $A_{2} B_{2} C_{2} D_{2}$ with the sides

$$
a_{2}=x, b_{2}=x, c_{2}=y, d_{2}=y .
$$

Theorem 3 In every bicentric quadrilateral ABCD the following inequalities are true:

$$
\begin{aligned}
2 r\left(r+\sqrt{4 R^{2}+r^{2}}\right) & \leq \min \{a b+c d, b c+a d\} \leq 4 r\left(\sqrt{4 R^{2}+r^{2}}-r\right) \\
& \leq \max \{a b+c d+b c+a d\} \leq 4 R^{2} .
\end{aligned}
$$


Proof. We suppose that $x_{1} \leq x_{2}, x_{1}+x_{2}=s^{2}, x_{1} x_{2}=\alpha s^{2}$ where

$$
\alpha=\frac{8 R^{2} r}{\sqrt{4 R^{2}+r^{2}}+r}=2 r\left(\sqrt{4 R^{2}+r^{2}}-r\right) .
$$

It results that: $x_{1}=\frac{s^{2}-\sqrt{s^{4}-4 \alpha s^{2}}}{2}, x_{2}=\frac{s^{2}+\sqrt{s^{4}-4 \alpha s^{2}}}{2}$. We consider the functions $f, g:(0,+\infty) \rightarrow R$.

$$
f(s)=\frac{s^{2}-\sqrt{s^{4}-4 \alpha s^{2}}}{2}, g(s)=\frac{s^{2}+\sqrt{s^{4}-4 \alpha s^{2}}}{2} .
$$

After differentiation we obtain:

$f^{\prime}(s)=\frac{s\left(\sqrt{s^{4}-4 \alpha s^{2}}-s^{2}+2 \alpha\right)}{\sqrt{s^{4}-4 \alpha s^{2}}} \leq 0, g^{\prime}(s)=\frac{s\left(\sqrt{s^{4}-4 \alpha s^{2}}+s^{2}-4 \alpha\right)}{\sqrt{s^{4}-4 \alpha s^{2}}} \geq 0$.

From Theorem 2 it results that: $s^{2} \geq 8 r\left(\sqrt{4 R^{2}+r^{2}}-r\right)=4 \alpha$.

It results that $f$ is a decreasing and $g$ is an increasing function. Because $s \leq r+\sqrt{4 R^{2}+r^{2}}$ we have $f\left(r+\sqrt{4 R^{2}+r^{2}}\right) \leq f(s)=x_{1}$. If follows that

$$
\begin{aligned}
& x_{1} \geq \frac{1}{2}\left[\left(r+\sqrt{4 R^{2}+r^{2}}\right)^{2}\right. \\
& \left.-\left(r+\sqrt{4 R^{2}+r^{2}}\right) \sqrt{\left(r+\sqrt{4 R^{2}+r^{2}}\right)^{2}-8 r\left(\sqrt{4 R^{2}+r^{2}}-r\right)}\right] \\
& =\frac{\left(r+\sqrt{4 R^{2}+r^{2}}\right)}{2}\left[r+\sqrt{4 R^{2}+r^{2}}\right. \\
& -\sqrt{r^{2}+4 R^{2}+r^{2}+2 r \sqrt{4 R^{2}+r^{2}}-8 r \sqrt{4 R^{2}+r^{2}}+8 r^{2}} \\
& =\frac{\left(r+\sqrt{4 R^{2}+r^{2}}\right)}{2}\left[r+\sqrt{4 R^{2}+r^{2}}-\sqrt{\left(\sqrt{4 R^{2}+r^{2}}\right)^{2}+9 r^{2}-6 r \sqrt{4 R^{2}+r^{2}}}\right] \\
& =2 r\left(r+\sqrt{4 R^{2}+r^{2}}\right) \text {. }
\end{aligned}
$$

It follows that

$$
x_{1} \geq 2 r\left(r+\sqrt{4 R^{2}+r^{2}}\right) .
$$


From $s \leq r+\sqrt{4 R^{2}+r^{2}}$ it results also that

$$
\begin{aligned}
x_{2}= & g(s) \leq g\left(r+\sqrt{4 R^{2}+r^{2}}\right) \\
= & \frac{1}{2}\left[\left(r+\sqrt{4 R^{2}+r^{2}}\right)^{2}\right. \\
& \left.+\left(r+\sqrt{4 R^{2}+r^{2}}\right) \sqrt{\left(r+\sqrt{4 R^{2}+r^{2}}\right)^{2}-8 r\left(\sqrt{4 R^{2}+r^{2}}-r\right)}\right] \\
= & \left(\sqrt{4 R^{2}+r^{2}}+r\right)\left(\sqrt{4 R^{2}+r^{2}}-r\right)=4 R^{2} .
\end{aligned}
$$

Thus we get the following inequality

$$
x_{2} \leq 4 R^{2}
$$

Since $8 r\left(\sqrt{4 R^{2}+r^{2}}-r\right) \leq s^{2}$ we have $x_{1}=f(s) \leq f\left(\sqrt{8 r\left(\sqrt{4 R^{2}+r^{2}}-r\right)}\right)$ or in an equivalent form

$$
\begin{aligned}
x_{1} \leq & \frac{1}{2}\left[8 r\left(\sqrt{4 R^{2}+r^{2}}-r\right)\right. \\
& \left.-\sqrt{8 r\left(\sqrt{4 R^{2}+r^{2}}-r\right)} \sqrt{8 r\left(\sqrt{4 R^{2}+r^{2}}-r\right)-8 r\left(\sqrt{4 R^{2}+r^{2}}-r\right)}\right] \\
= & 4 r\left(\sqrt{4 R^{2}+r^{2}}-r\right) .
\end{aligned}
$$

It follows that

$$
x_{1} \leq 4 r\left(\sqrt{4 R^{2}+r^{2}}-r\right) .
$$

Because $8 r\left(\sqrt{4 R^{2}+r^{2}}-r\right) \leq s^{2}$ and $g$ is an increasing function it follows that:

$$
g\left(\sqrt{8 r\left(\sqrt{4 R^{2}+r^{2}}-r\right)}\right) \leq g(s)=x_{2} \text { or } x_{2} \geq 4 r\left(\sqrt{4 R^{2}+r^{2}}-r\right) .
$$

From (7) (8) (9) and (10) it results that:

$$
x_{3}=2 r\left(r+\sqrt{4 R^{2}+r^{2}}\right) \leq x_{1} \leq 4 r\left(\sqrt{4 R^{2}+r^{2}}-r\right) \leq x_{2} \leq 4 R^{2} .
$$


Remark 1 From Theorem 3 it results that $2 \mathrm{r}\left(\mathrm{r}+\sqrt{4 \mathrm{R}^{2}+\mathrm{r}^{2}}\right) \leq 4 \mathrm{r}\left(\sqrt{4 \mathrm{R}^{2}+\mathrm{r}^{2}}-\right.$ $r)$ which, after performing some calculation, represent the well-known Fejes inequality $\mathrm{R} \geq \sqrt{2} \mathrm{r}$.

Theorem 4 In every bicentric quadrilateral ABCD the following inequalities are true:

$$
\begin{aligned}
\frac{r\left(\sqrt{4 R^{2}+r^{2}}+r\right)}{R} & \leq \min \left\{d_{1}, d_{2}\right\} \leq \frac{\sqrt{4 R^{2}+r^{2}}+r}{R} \sqrt{\frac{\left(\sqrt{4 R^{2}+r^{2}}-r\right) r}{2}} \\
& \leq \max \left\{d_{1}, d_{2}\right\} \leq 2 R .
\end{aligned}
$$

Proof. We suppose that $x_{1} \leq x_{2}$.

From Ptolemy's theorem it results that $\frac{x_{1}}{x_{2}}=\frac{d_{1}}{d_{2}}$ which implies $d_{1} \leq d_{2}$.

Because $d_{1} d_{2}=x_{3}$ we have

$$
\begin{aligned}
d_{1}^{2} & =\frac{x_{1}}{x_{2}} x_{3}=\frac{s^{2}-\sqrt{s^{4}-4 \alpha s^{2}}}{s^{2}+\sqrt{s^{4}-4 \alpha s^{2}}} x_{3}=x_{3} \frac{\left(s^{2}-\sqrt{s^{4}-4 \alpha s^{2}}\right)^{2}}{4 \alpha s^{2}} \\
& =x_{3} \frac{2 s^{4}-4 \alpha s^{2}-2 s^{2} \sqrt{s^{4}-4 \alpha s^{2}}}{4 \alpha s^{2}}=\frac{x_{3}\left(s^{2}-2 \alpha-\sqrt{s^{4}-4 \alpha s^{2}}\right)}{2 \alpha} \\
& =\frac{2 r\left(r+\sqrt{4 R^{2}+r^{2}}\right)}{4 r\left(\sqrt{4 R^{2}+r^{2}}-r\right)}\left[s^{2}-\sqrt{s^{4}-4 \alpha s^{2}}-2 \alpha\right] \\
& =\frac{\left(\sqrt{4 R^{2}+r^{2}}+r\right)^{2}}{8 R^{2}}\left[s^{2}-\sqrt{s^{4}-4 \alpha s^{2}}-2 \alpha\right]=B\left(2 x_{1}-2 \alpha\right),
\end{aligned}
$$

where we denote $B=\frac{\left(\sqrt{4 R^{2}+r^{2}}+r\right)^{2}}{8 R^{2}}$.

But from Theorem 3 we have

$$
4 r\left(r+\sqrt{4 R^{2}+r^{2}}\right) \leq 2 x_{1} \leq 8 r\left(\sqrt{4 R^{2}+r^{2}}-r\right) .
$$

We obtain

$$
\begin{aligned}
& 4 r\left(r+\sqrt{4 R^{2}+r^{2}}\right)-2 \alpha \leq 2 x_{1}-2 \alpha \leq 8 r\left(\sqrt{4 R^{2}+r^{2}}-r\right)-2 \alpha \text { or } \\
& 8 r^{2} \leq 2 x_{1}-2 \alpha \leq 4 r\left(\sqrt{4 R^{2}+r^{2}}-r\right) \text { or } \\
& 8 r^{2} B \leq B\left(2 x_{1}-2 \alpha\right) \leq 4 r\left(\sqrt{4 R^{2}+r^{2}}-r\right) B \text { or } \\
& \frac{8 r^{2}\left(\sqrt{4 R^{2}+r^{2}}+r\right)^{2}}{8 R^{2}} \leq d_{1}^{2} \leq \frac{4 r\left(\sqrt{4 R^{2}+r^{2}}-r\right)\left(\sqrt{4 R^{2}+r^{2}}+r\right)^{2}}{8 R^{2}} .
\end{aligned}
$$


It results that:

$$
\frac{r\left(\sqrt{4 R^{2}+r^{2}}+r\right)}{r}<d_{1} \leq \frac{\sqrt{4 R^{2}+r^{2}}+r}{R} \sqrt{\frac{\left(\sqrt{4 R^{2}+r^{2}}-r\right) r}{2}} .
$$

Also:

$$
\begin{aligned}
d_{2}^{2} & =\frac{x_{2}}{x_{1}} x_{3}=\frac{s^{2}+\sqrt{s^{4}-4 \alpha s^{2}}}{s^{2}-\sqrt{s^{4}-4 \alpha s^{2}}} x_{3}=\frac{\left(s^{2}+\sqrt{s^{4}-4 \alpha s^{2}}\right)^{2}}{4 \alpha s^{2}} x_{3} \\
& =\frac{x_{3}}{4 \alpha s^{2}}\left(2 s^{4}-4 \alpha s^{2}+2 s^{2} \sqrt{s^{4}-4 \alpha s^{2}}\right)=\frac{x_{3}}{2 \alpha}\left(s^{2}+\sqrt{s^{4}-4 \alpha s^{2}}-2 \alpha\right) \\
& =\frac{x_{3}}{2 \alpha}\left(2 x_{2}-2 \alpha\right)=\frac{\left(\sqrt{4 R^{2}+r^{2}}+r\right)^{2}\left(2 x_{2}-2 \alpha\right)}{8 R^{2}} .
\end{aligned}
$$

But we have proved that $4 r\left(\sqrt{4 R^{2}+r^{2}}-r\right) \leq x_{2} \leq 4 R$. It results that:

$$
\begin{aligned}
& 4 r\left(\sqrt{4 R^{2}+r^{2}}-r\right) \leq 2 x_{2}-2 \alpha \leq 2\left(4 R^{2}+2 r^{2}-2 r \sqrt{4 R^{2}+r^{2}}\right) \text { or } \\
& 4 r\left(\sqrt{4 R^{2}+r^{2}}-r\right)\left(\frac{\sqrt{4 R^{2}+r^{2}}+r}{2 \sqrt{2} R}\right)^{2} \leq d_{2}^{2} \\
& \quad \leq 2\left(\sqrt{4 R^{2}+r^{2}}-r\right)^{2} \frac{\left(\sqrt{4 R^{2}+r^{2}}+r\right)^{2}}{8 R^{2}} \text { or } \\
& \frac{\sqrt{4 R^{2}+r^{2}}+r}{R} \sqrt{\frac{\left(\sqrt{4 R^{2}+r^{2}}-r\right) r}{2} \leq d_{2} \leq 2 R .}
\end{aligned}
$$

From (11) and (12) it results the inequalities from the statement.

Theorem 5 Let be $\alpha, \beta \in \mathrm{R}$ so that $\mathrm{s} \leq \alpha \mathrm{R}+\beta \mathrm{r}$ is true in every bicentric quadrilateral $\mathrm{ABCD}$. Then $2 \mathrm{R}+(4-2 \sqrt{2}) \mathrm{r} \leq \alpha \mathrm{R}+\beta \mathrm{r}$ is true in every bicentric quadrilateral ABCD.

Proof. We consider the case of the square with the sides $a=b=c=d=1$. We have $2 \leq \alpha \frac{1}{\sqrt{2}}+\beta \frac{1}{2}$. It results that

$$
4 \leq \sqrt{2} \alpha+\beta
$$


If $a=b=1, c=d=0$ it results that $R=\frac{1}{2}, r=0$.

It follows that

$$
1 \leq \frac{\alpha}{2} \text { or } \alpha \geq 2
$$

We know that

$$
R \geq \sqrt{2} r .
$$

From (13), (14) and (15) it results that

$$
\begin{aligned}
(\alpha-2) R+(\beta-4+2 \sqrt{2}) r & \geq(\alpha-2) \sqrt{2} r+(\beta-4+2 \sqrt{2}) r \\
& =(\alpha \sqrt{2}+\beta-4) r \geq 0
\end{aligned}
$$

therefore

$$
\alpha R+\beta r \geq 2 R+(4-2 \sqrt{2}) r
$$

Theorem 6 In every bicentric quadrilateral the following inequality is true:

$$
s \leq 2 R+(4-2 \sqrt{2}) r .
$$

Proof. From the Theorem 1 we have $s \leq r+\sqrt{4 R^{2}+r^{2}}$. We denote $x=\frac{R}{r}$.

We prove that

$$
r+\sqrt{4 R^{2}+r^{2}} \leq 2 R+(4-2 \sqrt{2}) r
$$

or in an equivalent form

$$
\begin{aligned}
& 1+\sqrt{4 x^{2}+1} \leq 2 x+4-2 \sqrt{2} \text { or } \sqrt{4 x^{2}+1} \leq 2 x+3-2 \sqrt{2} \text { or } \\
& 1 \leq 4(3-2 \sqrt{2}) x+(3-2 \sqrt{2})^{2} \text { or } x \geq \frac{(-2+2 \sqrt{2})(4-2 \sqrt{2})}{4(3-2 \sqrt{2})} .
\end{aligned}
$$

After performing some calculation it results that $x \geq \sqrt{2}$ which represents just the Fejes's inequality [2].

Theorem 7 In every bicentric quadrilateral ABCD the following inequalities are true:

1) $4 r\left(3 \sqrt{4 R^{2}+r^{2}}-5 r\right) \leq a^{2}+b^{2}+c^{2}+d^{2} \leq 8 R^{2}$; 
2) $2 r \sqrt{8 r\left(\sqrt{4 R^{2}+r^{2}}-r\right)}\left(7 \sqrt{4 R^{2}+r^{2}}-9 r\right) \leq \sum a^{2} b \leq 8 R^{2}+2 r^{2}$;

3) $2 r\left(5 \sqrt{4 R^{2}+r^{2}}-3 r\right) \leq \sum a b \leq 4\left(R^{2}+r^{2}+r \sqrt{4 R^{2}+r^{2}}\right)$;

4) $32 r^{2} \sqrt{4 R^{2}+r^{2}}\left(\sqrt{4 R^{2}+r^{2}}-r\right) \leq \sum a^{2} b c$ $\leq 4 r \sqrt{4 R^{2}+r^{2}}\left(r+\sqrt{4 R^{2}+r^{2}}\right)^{2}$

5) $\left(2 r^{2}+2 r \sqrt{4 R^{2}+r^{2}}\right) \sqrt{8 r\left(\sqrt{4 R^{2}+r^{2}}-r\right)} \leq \sum a b c$ $\leq 2 r\left(r+\sqrt{4 R^{2}+r^{2}}\right)^{2}$.

Proof. We have $\sigma_{2}=s^{2}+\alpha, \sigma_{3}=s \alpha$ where $\alpha=2 r^{2}+2 r \sqrt{r^{2}+4 R^{2}}$.

1) $\sum a^{2}=(2 s)^{2}-2 \sigma_{2}=4 s^{2}-2 \sigma_{2}=4 s^{2}-2 s^{2}-4 r^{2}-4 r \sqrt{4 R^{2}+r^{2}}$.

It results that: $\sum a^{2}=2 s^{2}-4 r^{2}-4 r \sqrt{4 R^{2}+r^{2}}$.

From Theorem 2 we obtain

$$
4 r\left(3 \sqrt{4 R^{2}+r^{2}}-5 r\right) \leq a^{2}+b^{2}+c^{2}+d^{2} \leq 8 R^{2} .
$$

2) $a^{2} b=a b(2 s-b-c-d)=2 s a b-a b^{2}-a b c-a b d$ or $a^{2} b+a b^{2}=$ $2 s a b-a b c-a b d$.

It results that $\sum a^{2} b=2 s \sigma_{2}-3 \sigma_{3}=2 s^{3}-s \alpha=s\left(2 s^{2}-\alpha\right)$ which implies $\sum a^{2} b=s\left(2 s^{2}-\alpha\right)$. We consider the increasing function

$$
\begin{aligned}
& f:(0,+\infty) \rightarrow R, f(s)=2 s^{3}-s \alpha, \text { with } f^{\prime}(s)=6 s^{2}-\alpha \geq 0 \text { as } \\
& s^{2} \geq 8 r\left(\sqrt{4 R^{2}+r^{2}}-r\right) \geq \frac{\alpha}{6}=\frac{2 r^{2}+2 r \sqrt{r^{2}+4 R^{2}}}{6} .
\end{aligned}
$$

The last inequality may be written as:

$$
24 \sqrt{4 R^{2}+r^{2}}-24 r \geq r+\sqrt{4 R^{2}+r^{2}} \text { or } 23 \sqrt{4 R^{2}+r^{2}} \geq 25 r .
$$

But from inequality of Fejes it results that

$$
23 \sqrt{4 R^{2}+r^{2}} \geq 25 \sqrt{9 r^{2}}=75 r>25 r .
$$


From Theorem 2 it results that:

$$
\begin{aligned}
& \sqrt{8 r\left(\sqrt{4 R^{2}+r^{2}}-r\right)}\left(16\left(\sqrt{4 R^{2}+r^{2}}-r\right)-2 r^{2}-2 r \sqrt{4 R^{2}+r^{2}}\right) \\
& \leq \sum a^{2} b \leq\left(r+\sqrt{4 R^{2}+r^{2}}\right) \\
& \left(2 r^{2}+8 R^{2}+2 r^{2}+2 r \sqrt{4 R^{2}+r^{2}}-2 r^{2}-2 r \sqrt{4 R^{2}+r^{2}}\right)
\end{aligned}
$$

which is equivalent with the inequality from the statement.

3) $\sigma_{2}=\sum a b=s^{2}+\alpha$ or $8 r\left(\sqrt{4 R^{2}+r^{2}}-r\right)+2 r^{2}+2 r \sqrt{4 R^{2}+r^{2}} \leq \sum a b \leq$ $r^{2}+4 R^{2}+r^{2}+2 r \sqrt{4 R^{2}+r^{2}}+2 r^{2}+2 r \sqrt{4 R^{2}+r^{2}}$

which is equivalent with the inequality from the statement.

4) $a^{2} b c=a a b c=(2 s-b-c-d) a b c=2 s a b c-a b^{2} c-a b c^{2}-a b c d$ or $a^{2} b c+a b^{2} c+a b c^{2}=2 s a b c-a b c d$ or $\sum a^{2} b c=2 s \sigma_{3}-4 a b c d=2 s$ $s \alpha-4 s^{2} r^{2}$ or $\sum a^{2} b c=s^{2}\left(2 \alpha-4 r^{2}\right)=s^{2}\left(4 r^{2}+4 r \sqrt{4 R^{2}+r^{2}}-4 r^{2}\right)=$ $4 \mathrm{r} \sqrt{4 \mathrm{R}^{2}+\mathrm{r}^{2}} \mathrm{~s}^{2}$.

From Theorem 2 it results the inequality from the statement.

5) $\sum a b c=s \alpha$.

According to Theorem 2 it results the inequality from the statement.

Theorem 8 Let be $\alpha, \beta, \gamma \in R, \beta \geq 4$ so that $s^{2} \leq \alpha R^{2}+\beta R r+\gamma r^{2}$ is true in all bicentric quadrilateral. Then

$$
4 R^{2}+4 R r+(8-4 \sqrt{2}) r^{2} \leq \alpha R^{2}+\beta R r+\gamma r^{2}
$$

is true in all bicentric quadrilateral.

Proof. We consider the case of the bicentric quadrilateral with $a=b=c=$ $\mathrm{d}=1$. It results that $4 \leq \frac{\alpha}{2}+\frac{\beta}{2 \sqrt{2}}+\frac{\gamma}{4}$ or $16 \leq 2 \alpha+\sqrt{2} \beta+\gamma$.

In the case of $a=b=1, c=d=0$ it results that $R=\frac{1}{2}, r=0$ and $\alpha \geq 4$. But from inequality $R \geq \sqrt{2} r$ we have: 


$$
\begin{aligned}
& (\alpha-4) \mathrm{R}^{2}+(\beta-4) \mathrm{Rr}+(\gamma-8+4 \sqrt{2}) \mathrm{r}^{2} \\
& \geq(\alpha-4) 2 r^{2}+\sqrt{2}(\beta-4) r^{2}+(\gamma-8+4 \sqrt{2}) r^{2} \\
& \geq(\alpha-4) 2 r^{2}+\sqrt{2}(\beta-4) r^{2}+(\gamma-8+4 \sqrt{2}) r^{2} \\
& =(2 \alpha+\sqrt{2} \beta+\gamma-16) r^{2} \geq 0 .
\end{aligned}
$$

Theorem 9 In every bicentric quadrilateral ABCD the following inequality is true:

$$
s^{2} \leq 4 R^{2}+4 R r+(8-4 \sqrt{2}) r^{2}
$$

Proof. Since $s^{2} \leq\left(r+\sqrt{4 R^{2}+r^{2}}\right)^{2}$ it is sufficient to prove that:

$$
\begin{aligned}
& \left(\sqrt{4 x^{2}+1}+1\right)^{2} \leq 4 x^{2}+4 x+8-4 \sqrt{2} \text { or } \\
& 4 x^{2}+1+1+2 \sqrt{4 x^{2}+1} \leq 4 x^{2}+4 x+8-4 \sqrt{2} \text { or } \\
& 2 \sqrt{4 x^{2}+1} \leq 4 x+6-4 \sqrt{2} \text { or } \\
& \sqrt{4 x^{2}+1} \leq 2 x+3-2 \sqrt{2} \text { or } 4 x^{2}+1 \leq 4 x^{2}+(12-8 \sqrt{2}) x+(3-2 \sqrt{2})^{2} \text { or } \\
& x \geq \frac{(1-3+2 \sqrt{2})(1+3-2 \sqrt{2})}{4(3-2 \sqrt{2})}=\frac{(\sqrt{2}-1)(2-\sqrt{2})}{3-2 \sqrt{2}}=\sqrt{2} .
\end{aligned}
$$

Theorem 10 In every bicentric quadrilateral ABCD the following inequalities are true:

1) $\sum a b c \leq 8 R^{2} r+8 R r^{2}+(16-8 \sqrt{2}) r^{3}$

2) $\sum a b \leq 4\left[R^{2}+2 R r+(4-2 \sqrt{2}) r^{2}\right]$;

3) $\sum a^{2} b c \leq 32 R^{3} r+16 R r^{3}+(80-32 \sqrt{2}) R^{2} r^{2}+(32-16 \sqrt{2}) r^{4}$. 


\section{Proof.}

1) We proved that $\sum a b c \leq 2 r\left(r+\sqrt{4 R^{2}+r^{2}}\right)^{2}$, and

$$
\left(r+\sqrt{4 R^{2}+r^{2}}\right)^{2} \leq 4 R^{2}+4 R r+(8-4 \sqrt{2}) r^{2} .
$$

It results that

$$
\sum a b c \leq 2 r\left(4 R^{2}+4 R r+(8-4 \sqrt{2}) r^{2}\right) .
$$

2) Since $\sqrt{4 R^{2}+r^{2}} \leq 2 R+(3-2 \sqrt{2}) r$, from Theorem 7 3) it results that:

$$
\begin{aligned}
\sum a b & \leq 4\left(R^{2}+r^{2}+r \sqrt{4 R^{2}+r^{2}}\right) \\
& \leq 4\left[R^{2}+r^{2}+r(2 R+(3-2 \sqrt{2}) r)\right] \\
& =4\left[R^{2}+r^{2}+2 R r+(3-2 \sqrt{2}) r^{2}\right] \text { or } \\
\sum a b & \leq 4\left[R^{2}+2 R r+(4-2 \sqrt{2}) r^{2}\right] .
\end{aligned}
$$

3) From Theorem 7 4) it results that:

$$
\begin{aligned}
\sum a^{2} b c \leq & 4 r \sqrt{4 R^{2}+r^{2}}\left(r+\sqrt{4 R^{2}+r^{2}}\right)^{2} \\
= & 4 r \sqrt{4 R^{2}+r^{2}}\left(r^{2}+4 R^{2}+r^{2}+2 r \sqrt{4 R^{2}+r^{2}}\right) \\
= & 8 r \sqrt{4 R^{2}+r^{2}}\left(2 R^{2}+r^{2}+r \sqrt{4 R^{2}+r^{2}}\right) \\
= & \left(16 R^{2} r+8 r^{3}\right) \sqrt{4 R^{2}+r^{2}}+8 r^{2}\left(4 R^{2}+r^{2}\right) \\
\leq & \left(16 R^{2} r+8 r^{3}\right)[2 R+(3-2 \sqrt{2}) r]+32 R^{2} r^{2}+8 r^{4} \\
= & 32 R^{3} r+(48-32 \sqrt{2}) R^{2} r^{2}+16 R r^{3}+(24-16 \sqrt{2}) r^{4} \\
& +32 R^{2} r^{2}+8 r^{4},
\end{aligned}
$$

which is equivalent with the inequality from the statement.

Theorem 11 In every bicentric quadrilateral ABCD the following inequalities are true: 
1) $2 r \sqrt{8 r\left(\sqrt{4 R^{2}+r^{2}}-r\right)}\left(5 \sqrt{4 R^{2}+r^{2}}-11 r\right) \leq \sum a^{3}$

$$
\leq 2\left(r+\sqrt{4 R^{2}+r^{2}}\right)\left(4 R^{2}-r^{2}-r \sqrt{4 R^{2}+r^{2}}\right) ;
$$

2) $352 R^{2} r^{2}+208 r^{4}-240 r^{3} \sqrt{4 R^{2}+r^{2}}$

$$
\leq \sum a^{3} b \leq\left(r+\sqrt{4 R^{2}+r^{2}}\right)^{2}\left(8 R^{2}-4 r^{2}\right)
$$

\section{Proof.}

1) $a^{3}=a^{2}(2 s-b-c-d)=2 a^{2} s-a^{2} b-a^{2} c-a^{2} d$ or $\sum a^{3}=2 s \sum a^{2}-$ $\sum a^{2} b=2 s\left(2 s^{2}-2 \alpha\right)-2 s^{3}+s \alpha$.

It results that $\sum a^{3}=2 s^{3}-3 \alpha s$.

We consider the function $f:(0,+\infty) \rightarrow R, f(s)=2 s^{3}-3 \alpha s$, with the derivate $f^{\prime}(s)=6 s^{2}-3 \alpha$. We prove that $f^{\prime}(s) \geq 0$ or $s^{2} \geq \frac{\alpha}{2}$.

But $s^{2} \geq 8 r\left(\sqrt{4 R^{2}+r^{2}}-r\right)$. It will be sufficient to prove that:

$$
\begin{aligned}
& 8 r\left(\sqrt{4 R^{2}+r^{2}}-r\right) \geq r^{2}+r \sqrt{4 R^{2}+r^{2}} \text { or } \\
& 8 \sqrt{4 x^{2}+1}-8 \geq 1+\sqrt{4 x^{2}+1} \text { or } \sqrt{4 x^{2}+1} \geq \frac{9}{7},
\end{aligned}
$$

which is true because $\sqrt{4 x^{2}+1} \geq 2$ according to Fejes inequality.

Since $f$ is an increasing function it results from Theorem 2 that:

$$
\begin{aligned}
& \sqrt{8 r\left(\sqrt{4 R^{2}+r^{2}}-r\right)}\left[16\left(\sqrt{4 R^{2}+r^{2}}-r\right)-6 r^{2}-6 r \sqrt{4 R^{2}+r^{2}}\right] \\
& \leq \sum a^{3} \leq\left(r+\sqrt{4 R^{2}+r^{2}}\right)\left[2 r^{2}+8 R^{2}+2 r^{2}\right. \\
& \left.+4 r \sqrt{4 R^{2}+r^{2}}-6 r^{2}-6 r \sqrt{4 R^{2}+r^{2}}\right],
\end{aligned}
$$

which is equivalent with the inequality from the statement.

2) $a^{3} b=a b\left(\sum a^{2}-b^{2}-c^{2}-d^{2}\right)=a b \sum a^{2}-a b^{3}-a b c^{2}-a b d^{2}$ or $a^{3} b+a b^{3}=a b \sum a^{2}-a b c^{2}-a b d^{2}$ or $\sum a^{3} b=\sum a b \sum a^{2}-\sum a^{2} b c=$ $\left(s^{2}+\alpha\right)\left(2 s^{2}-2 \alpha\right)-\left(2 \alpha-4 r^{2}\right) s^{2}$ or $\sum a^{3} b=2 s^{4}-\left(2 \alpha-4 r^{2}\right) s^{2}-2 \alpha^{2}$.

We denote $s^{2}=t$ and consider the function: $f:(0,+\infty) \rightarrow R$,

$$
f(t)=2 t^{2}-\left(2 a-4 r^{2}\right) t-2 a^{2}
$$


and

$$
t_{v}=\frac{2 a-4 r^{2}}{4}=\frac{a-2 r^{2}}{2}=r \sqrt{4 R^{2}+r^{2}} .
$$

We prove that $t \geq t_{v}$.

$s^{2} \geq r \sqrt{4 R^{2}+r^{2}}$. But $s^{2} \geq 8 r\left(\sqrt{4 R^{2}+r^{2}}-r\right)$. It will be sufficient to prove that

$$
8 r\left(\sqrt{4 R^{2}+r^{2}}-r^{2}\right) \geq r \sqrt{4 R^{2}+r^{2}} \text { or } \sqrt{4 R^{2}+r^{2}} \geq \frac{8}{7}
$$

which is true because $\sqrt{4 R^{2}+r^{2}} \geq 3$.

It results that $f$ is an increasing function which implies:

$$
\begin{aligned}
& 128 r^{2}\left(4 R^{2}+2 r^{2}-2 r \sqrt{4 R^{2}+r^{2}}\right)-4 r \sqrt{4 R^{2}+r^{2}} 8 r\left(\sqrt{4 R^{2}+r^{2}}-r\right) \\
& -2\left(2 r^{2}+2 r \sqrt{4 R^{2}+r^{2}}\right)^{2} \leq \sum a^{3} b \leq 2\left(r+\sqrt{4 R^{2}+r^{2}}\right)^{4} \\
& -4 r \sqrt{4 R^{2}+r^{2}}\left(r+\sqrt{4 R^{2}+r^{2}}\right)^{2}-2\left(2 r^{2}+2 r \sqrt{4 R^{2}+r^{2}}\right)^{2}
\end{aligned}
$$

or

$$
\begin{aligned}
& 512 R^{2} r^{2}+256 r^{4}-256 r^{3} \sqrt{4 R^{2}+r^{2}}-32 r^{2}\left(4 R^{2}+r^{2}\right)+32 r^{3} \sqrt{4 R^{2}+r^{2}} \\
& -8 r^{4}-8 r^{2}\left(4 R^{2}+r^{2}\right)-16 r^{3} \sqrt{4 R^{2}+r^{2}} \leq \sum a^{3} b \leq 2\left(r+\sqrt{4 R^{2}+r^{2}}\right)^{2} \\
& \left(r^{2}+4 R^{2}+r^{2}+2 r \sqrt{4 R^{2}+r^{2}}-2 r \sqrt{4 R^{2}+r^{2}}\right)-8 r^{2}\left(r+\sqrt{4 R^{2}+r^{2}}\right)^{2}
\end{aligned}
$$

or

$$
\begin{aligned}
352 R^{2} r^{2}+208 r^{4}-240 r^{3} \sqrt{4 R^{2}+r^{2}} \leq & \sum a^{3} b \leq\left(r+\sqrt{4 R^{2}+r^{2}}\right)^{2} \\
& \left(4 r^{2}+8 R^{2}-8 r^{2}\right) .
\end{aligned}
$$

Theorem 12 In every bicentric quadrilateral ABCD the following inequalities are true:
1) $\sum a^{3} \leq 16 R^{3}+(24-16 \sqrt{2}) R^{2} r-8 R r^{2}-(16-8 \sqrt{2}) r^{3}$;
2) $\sum a^{3} b \leq 32 R^{4}-16 R^{2} r^{2}+32 R^{3} r+16 R r^{3}+(64-32 \sqrt{2}) R^{2} r^{2}-(32-16 \sqrt{2}) r^{4}$; 
3) $\sum a^{3} b \geq 352 R^{2} r^{2}+(480 \sqrt{2}-512) r^{4}-480 R r^{3}$.

\section{Proof.}

1) From Theorem 11 it results that:

$$
\begin{aligned}
\sum a^{3} \leq & \left(r+\sqrt{4 R^{2}+r^{2}}\right)\left(8 R^{2}-2 r^{2}-2 r \sqrt{4 R^{2}+r^{2}}\right) \\
= & 8 R^{2} r-2 r^{3}-2 r^{2} \sqrt{4 R^{2}+r^{2}}+8 R^{2} \sqrt{4 R^{2}+r^{2}} \\
& -2 r^{2} \sqrt{4 R^{2}+r^{2}}-8 R^{2} r-2 r^{3} \\
= & \left(8 R^{2}-4 r^{2}\right) \sqrt{4 R^{2}+r^{2}}-4 r^{3} \\
\leq & \left(8 R^{2}-4 r^{2}\right)[2 R+(3-2 \sqrt{2}) r]-4 r^{3} \\
= & 16 r^{3}+(24-16 \sqrt{2}) R^{2} r-8 R r^{2}-(12-8 \sqrt{2}) r^{3}-4 r^{3},
\end{aligned}
$$

which is equivalent with inequality from the statement.

2) From Theorem 11 it results that

$$
\sum a^{3} b \leq\left(r+\sqrt{4 R^{2}+r^{2}}\right)^{2}\left(8 R^{2}-4 r^{2}\right)
$$

and

$$
\left(r+\sqrt{4 R^{2}+r^{2}}\right)^{2} \leq 4 R^{2}+4 R r+(8-4 \sqrt{2}) r^{2} .
$$

It results that:

$$
\begin{aligned}
\sum a^{3} b \leq & {\left[4 R^{2}+4 R r+(8-4 \sqrt{2}) r^{2}\right]\left(8 R^{2}-4 r^{2}\right) } \\
= & 32 R^{4}-16 R^{2} r^{2}+32 R^{3} r-16 R r^{3}+(64-32 \sqrt{2}) R^{2} r^{2} \\
& -(32-16 \sqrt{2}) r^{4},
\end{aligned}
$$

which is equivalent with the inequality from the statement.

3) We prove that:

$$
\begin{aligned}
\sum a^{3} b & \geq 352 R^{2} r^{2}+208 r^{4}-240 r^{3} \sqrt{4 R^{2}+r^{2}} \\
& \geq 352 R^{2} r^{2}+208 r^{4}-240 r^{3}[2 R+(3-2 \sqrt{2}) r] \\
& =352 R^{2} r^{2}+208 r^{4}-480 R r^{3}-(720-480 \sqrt{2}) r^{4}
\end{aligned}
$$

which is equivalent with the inequality from the statement. 


\section{References}

[1] W. J. Blundon, R. H. Eddy, Problem 488, Nieuw Arch. Wiskunde, 26 (1978).

[2] L. Fejes-Tóth, Inequalities concerning poligons and polyedra, Duke Math. J., 15 (1948), 817-822.

[3] T. Ovidiu, N. Pop Minculete, M. Bencze, An introduction to quadrilateral geometry, Editura Didactică şi Pedagogică, Bucureşti, 2013.

[4] Octogon Mathematical Magazine (1993-2013)

Received: 25 September 2013 